ARTICLE

\title{
2D atomic crystal molecular superlattices by soft plasma intercalation
}

Lufang Zhang ${ }^{1,7}$, Haiyan Nan (10 1,7, Xiumei Zhang ${ }^{2}$, Qifeng Liang ${ }^{3}$, Aijun Du ${ }^{4}$, Zhenhua $\mathrm{Ni}^{5}$, Xiaofeng Gu (D) ${ }^{1}$, Kostya (Ken) Ostrikov ${ }^{4,6} \&$ Shaoqing Xiao (10 ${ }^{1 \times}$

Two-dimensional (2D) atomic crystal superlattices integrate diverse 2D layered materials enabling adjustable electronic and optical properties. However, tunability of the interlayer gap and interactions remain challenging. Here we report a solution based on soft oxygen plasma intercalation. 2D atomic crystal molecular superlattices (ACMSs) are produced by intercalating $\mathrm{O}_{2}+$ ions into the interlayer space using the plasma electric field. Stable molecular oxygen layer is formed by van der Waals interactions with adjacent transition metal dichalcogenide (TMD) monolayers. The resulting interlayer gap expansion can effectively isolate TMD monolayers and impart exotic properties to homo- $\left(\mathrm{MoS}_{2}\left[\mathrm{O}_{2}\right]_{x}\right)$ and hetero$\left(\mathrm{MoS}_{2}\left[\mathrm{O}_{2}\right]_{x} / \mathrm{WS}_{2}\left[\mathrm{O}_{2}\right]_{x}\right)$ stacked ACMSs beyond typical capacities of monolayer TMDs, such as 100 times stronger photoluminescence and 100 times higher photocurrent. Our potentially universal approach to tune interlayer stacking and interactions in 2D ACMSs may lead to exotic superlattice properties intrinsic to monolayer materials such as direct bandgap pursued for future optoelectronics.

\footnotetext{
${ }^{1}$ Engineering Research Center of IoT Technology Applications (Ministry of Education), Department of Electronic Engineering, Jiangnan University, Wuxi 214122, China. ${ }^{2}$ School of Science, Jiangnan University, Wuxi 214122, China. ${ }^{3}$ Department of Physics, Shaoxing University, Shaoxing 312000, China. ${ }^{4}$ School of Chemistry and Physics and QUT Centre for Materials Science, Queensland University of Technology (QUT), Brisbane QLD 4000, Australia. ${ }^{5}$ Department of Physics and Key Laboratory of MEMS of the Ministry of Education, Southeast University, Nanjing 211189, China. ${ }^{6}$ CSIRO-QUT Joint Sustainable Processes and Devices Laboratory, P.O. Box 218, Lindfield, NSW 2070, Australia. ${ }^{7}$ These authors contributed equally: Lufang Zhang, Haiyan Nan.

凶email: xiaosq@jiangnan.edu.cn
} 
T wo-dimensional (2D) atomic crystal superlattices allow effective manipulation of stacking and coupling of atomic layers of diverse materials without restricting lattice matching due to the van der Waals forces between the adjacent layers. Therefore, such artificial $2 \mathrm{D}$ atomic crystal superlattices possess a wide range of adjustable electronic properties, offering technological opportunities, and applications beyond the reach of existing materials $\mathrm{s}^{1-5}$. The most common fabrication methods based on layer-by-layer exfoliation and transfer are complex, laborious, and time-consuming, with limited yield and reproducibility $^{6-11}$. Chemical vapor deposition (CVD) has been successfully applied to produce high-quality $2 \mathrm{D}$ heterostructures, bilayer, and multilayer transition metal dichalcogenides (TMDs), as well as lateral surface superlattices. However, CVD lacks growth and stacking precision and is thus impractical for highorder vertical superlattices ${ }^{12-17}$.

Recently, a new approach for the formation of vertical 2D superlattices has been demonstrated. Instead of growing or stacking layers of different 2D materials on top of each other, superlattice structures can be produced by the intercalation of selected 2D atomic crystals with alkali metal ions via electrochemical reactions ${ }^{18-20}$. Molecular intercalation is now regarded as a new and promising way to create the new class of stable superlattices in which monolayer atomic crystals alternate with ammonium bromide molecular layers ${ }^{4}$. However, the current state-of-the-art electrochemical approach is a wet process that often uses a whole 2D crystal as the source material and thus suffers from excessive material consumption. Furthermore, the alkali metal intercalation method usually requires the protection of inert gas to avoid the degradation of the properties of these superlattices, while the ammonium bromide molecule used for electrochemical intercalation is a toxic material.

To address the above issues, here we propose a soft oxygen plasma intercalation concept and demonstrate the $2 \mathrm{D}$ atomic crystal molecular superlattices where monolayer TMDs alternate with oxygen molecular layers. This dry method is effective for both mechanically exfoliated or CVD-grown TMD flakes (including $\mathrm{MoS}_{2}, \mathrm{WS}_{2}, \mathrm{MoSe}_{2}, \mathrm{WSe}_{2}$, and $\mathrm{ReS}_{2}$, etc.) with thicknesses ranging from 2 to 8 layers.

By using $\mathrm{MoS}_{2}$ as a model system, we demonstrate that plasma intercalation with oxygen molecular layers produces $\operatorname{MoS}_{2}\left[\mathrm{O}_{2}\right]_{x}$ superlattices in which the interlayer distance increases from 0.6 to $0.9 \mathrm{~nm}$ compared to pure $\mathrm{MoS}_{2}$, thereby effectively decoupling the $\mathrm{MoS}_{2}$ monolayers. As such, the $\mathrm{MoS}_{2}\left[\mathrm{O}_{2}\right]_{x}$ superlattices display extremely strong photoluminescence (PL) with an intensity approximately 100 times higher compared to pristine $\mathrm{MoS}_{2}$. We confirm the superlattice structure by means of PL spectroscopy, Raman spectroscopy, atomic force microscopy (AFM), X-ray photoelectron spectroscopy (XPS), transmission electron microscopy (TEM) as well as first-principle atomistic numerical simulations. The bilayer $\operatorname{MoS}_{2}\left[\mathrm{O}_{2}\right]_{x} / \mathrm{WS}_{2}\left[\mathrm{O}_{2}\right]_{x}$ superlattice lateral heterostructures show much better photoelectric performance than the pristine bilayer $\mathrm{MoS}_{2} / \mathrm{WS}_{2}$ lateral heterostructures.

\section{Results}

The schematic and chemical reaction of soft plasma intercalation. Figure 1 shows the schematic of fabricating monolayer$\mathrm{MoS}_{2} / \mathrm{O}_{2}$-molecule superlattices by the soft oxygen plasma intercalation. The soft oxygen plasma was excited in the capacitive discharge mode (E-mode) of a planar low-frequency $(2 \mathrm{MHz})$ inductively coupled plasma system, which is sketched in Supplementary Fig. 1. The capacitive coupling originating from the radial potential drop across the two ends of the planar induction coil generates the radial electrostatic field parallel to the substrate surface. This electrostatic field drives positive oxygen ions along

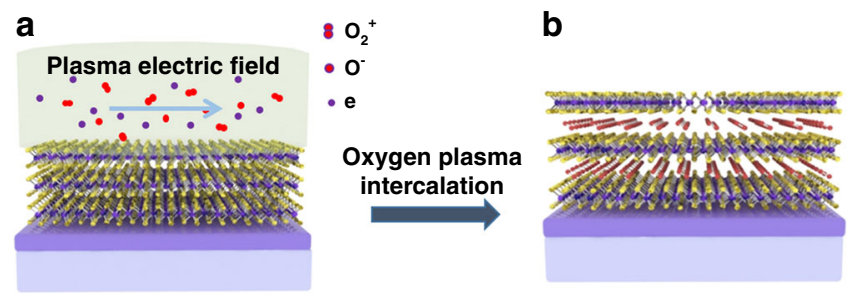

Fig. 1 Soft oxygen plasma intercalation creates 2D ACMSs. a Schematic of the soft oxygen plasma treatment of few-layer $\mathrm{MoS}_{2}$ flakes, where the plasma-induced radial electrostatic field is parallel to the interlayer space of $\mathrm{MoS}_{2}$ flakes, and the oxygen plasma contains $\mathrm{O}_{2}+$ ions, $\mathrm{O}^{-}$ions, $\mathrm{O}_{2}$ molecules, and electrons. b $\mathrm{MoS}_{2}\left[\mathrm{O}_{2}\right]_{x}$ superlattices arise through the plasma-enabled oxygen intercalation and formation of the oxygen molecular layer within the interlayer spaces of the few-layer $\mathrm{MoS}_{2}$ flakes.

the substrate surface and facilitates oxygen intercalation into the interlayer space between every two adjacent TMD layers. Furthermore, the E-mode discharge usually operates at very low input power $(5-30 \mathrm{~W})$ so that the ion density is too low to induce damage to the treated flakes. The chemical reaction of the soft oxygen plasma intercalation process consists of two half-reactions:

$$
\begin{gathered}
3 \mathrm{O}_{2}+\mathrm{e} \stackrel{\text { ionization }}{\rightarrow} 2 \mathrm{O}_{2}^{+}+2 \mathrm{O}^{-}+\mathrm{e}, \\
\mathrm{MoS}_{2}+x \mathrm{O}_{2}^{+}+x \mathrm{e} \stackrel{\text { intercalation }}{\longrightarrow} \mathrm{MoS}_{2}\left[\mathrm{O}_{2}\right]_{x} .
\end{gathered}
$$

The oxygen molecules formed in the interlayer space after oxygen ion intercalation may be stabilized via the van der Waals interaction with the adjacent $\mathrm{MoS}_{2}$ monolayers thereby forming the $\mathrm{MoS}_{2}\left[\mathrm{O}_{2}\right]_{x}$ superlattices.

Microscopic and optical characterization of $\mathrm{MoS}_{2}\left[\mathrm{O}_{2}\right]_{x}$ superlattices. Figure $2 \mathrm{a}, \mathrm{b}$ displays the AFM images together with the corresponding optical images of a mechanically exfoliated $\mathrm{MoS}_{2}$ flake taken before and after $3 \mathrm{~min}$ of the oxygen plasma intercalation, respectively. AFM images reveal an apparent increase in the cross-sectional thickness from 2.54 to $3.72 \mathrm{~nm}$. The slight change of the root-mean-square (RMS) roughness from $0.46 \pm$ $0.14 \mathrm{~nm}$ to $0.74 \pm 0.10 \mathrm{~nm}$ suggests that the surface of the sample was less affected by the $3 \mathrm{~min}$ oxygen plasma intercalation. The $1.18 \mathrm{~nm}$ increase in thickness, distributed across the $2.54 \mathrm{~nm}$ $\mathrm{MoS}_{2}$ flake consisting of 4 monolayers by assuming $0.65 \mathrm{~nm}$ as the thickness of a single S-Mo-S layer, corresponds to an average increase in each van der Waals gap of $3.93 \AA$. Further studies using TEM (Fig. 2c, d) show the distinct difference in microstructure between the pristine and treated flakes, giving a clearly resolved interlayer distance expansion ranging from $6.05 \AA$ in the pristine sample (Fig. 2c) to $9.27 \AA$ in the treated one (Fig. 2d). The deduced interlayer distance expansion $(3.22 \AA)$ is consistent with that $(3.93 \AA)$ obtained by the AFM measurements within the error margins, further validating the formation of $\mathrm{MoS}_{2}\left[\mathrm{O}_{2}\right]_{x}$ superlattices.

More interestingly, as shown in Fig. 2e, the PL intensity of the $\mathrm{MoS}_{2}$ flake can be strongly enhanced by 100-fold after the oxygen plasma intercalation, along with a $\sim 32 \mathrm{meV}$ decrease of the full width at half-maximum (FWHM). For the pristine few-layer $\mathrm{MoS}_{2}$, the PL peak is well fitted to two peaks by using Lorentzian functions, which are assigned to $\mathrm{A}$ exciton $(\sim 1.83 \mathrm{eV})$ and $\mathrm{B}$ exciton $(\sim 1.96 \mathrm{eV})$, respectively ${ }^{21,22}$. Specifically, peak $\mathrm{A}$ is due to the direct electron-hole recombination in a neutral exciton at the $\mathrm{K}$ point, while peak $\mathrm{B}$ with higher energy is ascribed to the indirect electron-hole recombination in a neutral exciton at a lower valence band because of the spin-orbit coupling. 

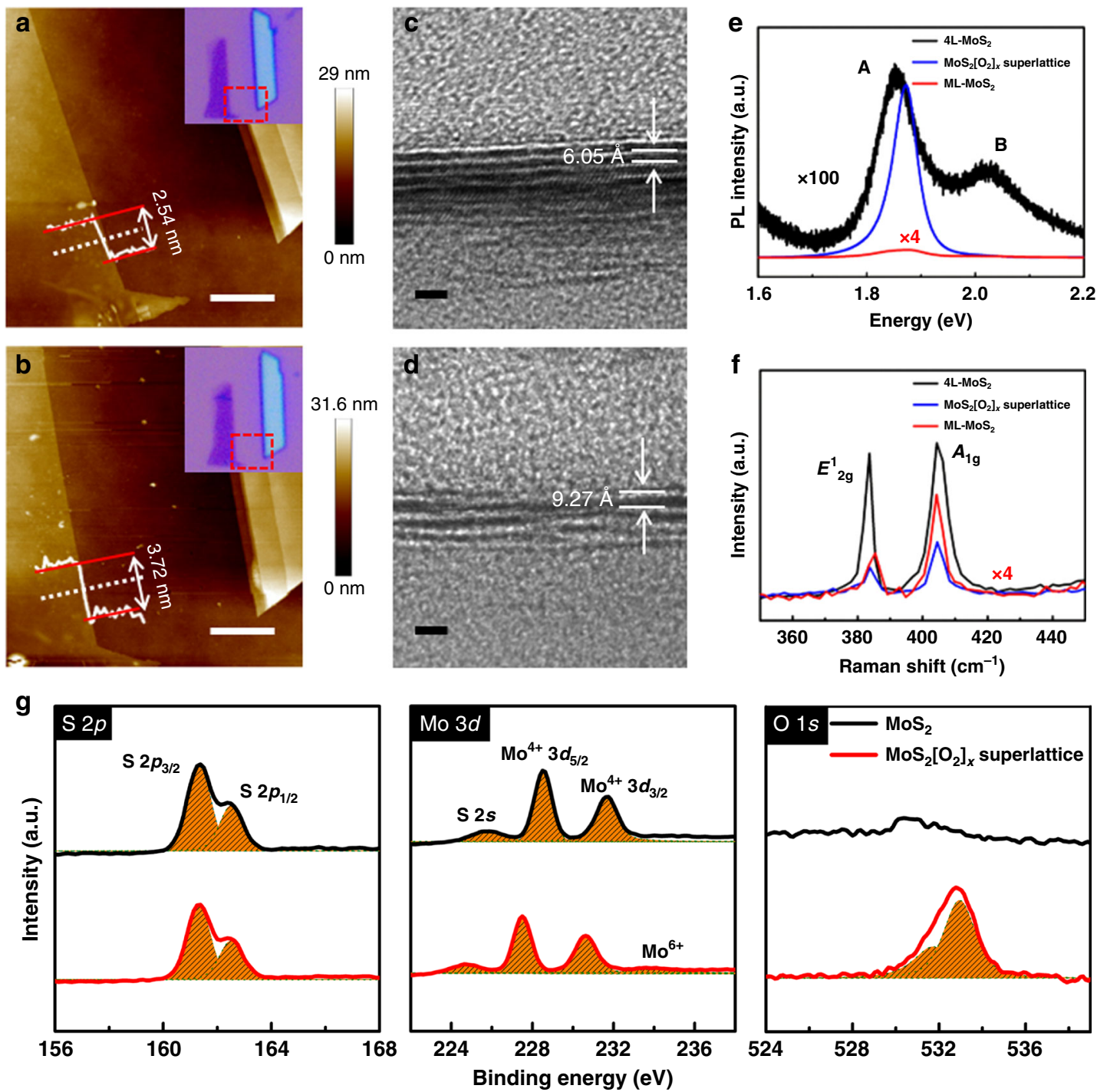

Fig. 2 Structure and property evolution from $\mathbf{M o S}_{\mathbf{2}}$ flake to $\mathbf{M o S 2}[\mathbf{0 2}]_{\mathbf{x}}$ superlattice. a, b AFM images of a pristine mechanically exfoliated four-layer $\mathrm{MoS}_{2}$ flake and the corresponding $\mathrm{MoS}_{2}\left[\mathrm{O}_{2}\right]_{x}$ superlattice obtained by the 3 min long oxygen plasma intercalation. Insets are the corresponding optical images and height profiles along the dashed white lines. Scale bars: $1 \mu \mathrm{m}$. c, d Cross-sectional TEM images of a pristine $\mathrm{MoS}_{2}$ flake and a $\mathrm{MoS}_{2}\left[\mathrm{O}_{2}\right]_{x}$ superlattice. Scale bars: $2 \mathrm{~nm}$. e, $\mathbf{f} \mathrm{PL}$ and Raman spectra of the $\mathrm{MoS}_{2}$ flake and $\mathrm{MoS}_{2}\left[\mathrm{O}_{2}\right]_{x}$ superlattice in a and $\mathbf{b}$, respectively, as well as the corresponding spectra (after multiplying by four) of monolayer $\mathrm{MoS}_{2}$. $\mathbf{g}$ High-resolution XPS spectra of S 2p, Mo $3 d$, and $\mathrm{O} 1 \mathrm{~s}$ of the pristine MoS flake and $\mathrm{MoS}_{2}\left[\mathrm{O}_{2}\right]_{x}$ superlattice.

For the $\operatorname{MoS}_{2}\left[\mathrm{O}_{2}\right]_{x}$ superlattice, however, the intensity of peak A is greatly enhanced by 100 -fold while peak B vanishes. It is well-known that the layer number reduction from multilayer to monolayer can also lead to this variation in PL properties due to the indirect-to-direct bandgap transition ${ }^{23}$. The frequency differences between $E_{2 \mathrm{~g}}^{1}$ (in-plane vibrational mode) and $A_{1 \mathrm{~g}}$ (out-plane vibrational mode) peak of Raman spectra provide a reliable means to determine the number of layers ${ }^{24,25}$. The Raman spectra in Fig. $2 \mathrm{f}$ show a slight change of the frequency difference between $E_{2 \mathrm{~g}}^{1}$ and $A_{1 \mathrm{~g}}$ modes from 23.6 to $23.0 \mathrm{~cm}^{-1}$, indicating that the number of layers in the 4-layer $(4 \mathrm{~L})$ flake does not change. Both the decrease in Raman intensity of $E_{2 g}^{1}$ mode and the disorder between some of the layers in the TEM image (Fig. 2d) may be attributed to the disorder introduced in the $\mathrm{MoS}_{2}$ lattice by the incorporation of a small amount of oxygencontaining species as validated by the following XPS and firstprinciple calculation analysis. Another detailed experiment on the 6-layer $\mathrm{MoS}_{2}$ flake (Supplementary Fig. 2) reveals a thickness increase of $1.8 \mathrm{~nm}$ (corresponding to an average increase in each van der Waals gap of $3 \AA$ ), a PL enhancement of 60 -fold (with peak A greatly enhanced and peak B disappearing) as well as similar Raman spectra behaviors.

We emphasize that both the astonishing enhancement of peak A and the vanishment of peak B are not the result of the indirectto-direct bandgap transition from multilayer to monolayer due to a reduction in the layer numbers as proved by AFM, TEM, and Raman measurements. Instead, the observed indirect-to-direct bandgap transition owes to the formation of $\mathrm{MoS}_{2}\left[\mathrm{O}_{2}\right]_{x}$ superlattices which can effectively isolate the $\mathrm{MoS}_{2}$ monolayers ${ }^{26,27}$.

In order to testify the universality of our soft oxygen plasma intercalation on $\mathrm{MoS}_{2}$ flakes, we performed similar experiments on $32 \mathrm{MoS}_{2}$ flakes with different layer numbers ranging from 2 to 8 layers and list PL (including peak A position shift, FWHM, and intensity enhancement) and Raman (including .. position and $A_{1 \mathrm{~g}}$ position, FWHM) properties in Supplementary Tables 1 and 2, respectively. On average, the PL intensity (peak A) is increased by 53.1 times, and the FWHM of peak A decreases by $31 \mathrm{meV}$. 
Furthermore, the Raman statistical data shows that $E_{2 \mathrm{~g}}^{1}$ position hardly changes while $A_{1 \mathrm{~g}}$ position has a slight redshift of $0.7 \mathrm{~cm}^{-1}$ on average, revealing that the intralayer coupling is unaffected by the plasma intercalation, while the interlayer van der Waals coupling (out-of-plane vibration mode) becomes significantly weaker due to the isolation of every two adjacent $\mathrm{MoS}_{2}$ monolayers by the intercalated oxygen molecular layer. However, the van der Waals coupling between adjacent layers still exists and the corresponding Raman signals $\left(A_{1 \mathrm{~g}}\right)$ can reflect the multilayer property as observed. On the other hand, the largely reduced van der Waals coupling plays a negligible effect on the PL effect as proved by the following calculated energy bands and therefore such $\operatorname{MoS}_{2}\left[\mathrm{O}_{2}\right]_{x}$ superlattices exhibit an extremely strong PL behavior similar to that of $\mathrm{MoS}_{2}$ monolayer.

We also used energy-dependent XPS to probe the oxygen intercalation effect by comparing high-resolution XPS spectra of $\mathrm{S}$ $2 p$, Mo $3 d$, and $\mathrm{O} 1 s$ between the pristine $\mathrm{MoS}_{2}$ flake and the $\mathrm{MoS}_{2}\left[\mathrm{O}_{2}\right]_{x}$ superlattice, as shown in Fig. $2 \mathrm{~g}$. The strong peaks at 161.5 and $162.4 \mathrm{eV}$ corresponding to $\mathrm{S} 2 p_{3 / 2}$ and $\mathrm{S} 2 p_{1 / 2}$ states, respectively, hardly change without any new peak emerging, indicating no $\mathrm{S}-\mathrm{O}$ bonds formed during the oxygen plasma intercalation $^{28}$. For O $1 s$ and Mo $3 d$, however, the emergence of a strong peak at around $532 \mathrm{eV}$ and a very small peak at $233.3 \mathrm{eV}$ (apart from prominent $\mathrm{Mo}^{4+} 3 d_{3 / 2}$ and $\mathrm{Mo}^{4+} 3 d_{5 / 2}$ at 213.8 and $228.5 \mathrm{eV}$ ), respectively, reveal the fundamental change in microstructure after the oxygen plasma intercalation. The newly emerging $\mathrm{O} 1 \mathrm{~s}$ peak can be decomposed into a peak at $531.7 \mathrm{eV}$ $\left(\mathrm{MoO}_{3}\right)$ and another peak at $532.9 \mathrm{eV}\left(\mathrm{O}_{2} \text { molecule }\right)^{29}$, while the small Mo $3 d$ peak at $233.3 \mathrm{eV}$ can be ascribed to $\mathrm{Mo}^{6+}\left(\mathrm{MoO}_{3}\right)^{30}$. In addition, doublet Mo $3 d$ peaks also present a shift toward lower binding energy which indicates a shift in the Femi level toward the valence band and further validates the $\mathrm{p}$-type doping by oxygen substitution at sulfur vacancies ${ }^{31,32}$. For the $\operatorname{MoS}_{2}\left[\mathrm{O}_{2}\right]_{x}$ superlattice, the atomic percentage of $\mathrm{O}$ in the chemical form of $\mathrm{MoO}_{3}$ and $\mathrm{O}_{2}$ is estimated to be $0.348: 0.652$, meaning that most oxygen elements exist in $\mathrm{O}_{2}$ molecules and interact with $\mathrm{MoS}_{2}$ monolayer via van der Waals coupling to construct the superlattice in which $\mathrm{MoS}_{2}$ monolayers alternate with oxygen molecular layers.

Mechanism of the soft oxygen plasma intercalation process. In order to exclude potentially competing for oxygen bonding mechanisms that may expand the interlayer space, we carried out density functional theory (DFT) calculations on pristine $\mathrm{MoS}_{2}$ bilayer and five oxygen-incorporated $\mathrm{MoS}_{2}$ bilayer systems, namely $\mathrm{MoS}_{2}$ bilayer with substitutional $\mathrm{O}$ at $\mathrm{S}$ site (Os), with substitutional $2 \mathrm{O}$ at $2 \mathrm{~S}$ sites (2Os), with oxygen molecule layer intercalated $\left(\mathrm{MoS}_{2}\left[\mathrm{O}_{2}\right]_{x}\right)$, with both oxygen molecule layer intercalated and substitutional $\mathrm{O}$ site at $\mathrm{S}$ site $\left(\mathrm{MoS}_{2}\left[\mathrm{O}_{2}\right]_{x}+\mathrm{O}_{\mathrm{S}}\right)$, with both oxygen molecule layer intercalated and substitutional $2 \mathrm{O}$ site at $2 \mathrm{~S}$ sites $\left(\mathrm{MoS}_{2}\left[\mathrm{O}_{2}\right]_{x}+2 \mathrm{O}_{\mathrm{S}}\right)$, as shown in Fig. 3. For the $\mathrm{MoS}_{2}\left[\mathrm{O}_{2}\right]_{x}+\mathrm{O}_{\mathrm{S}}$ structure, the atomic percentage of $\mathrm{O}$ in the chemical form of $\mathrm{MoO}_{3}$ and $\mathrm{O}_{2}$ is $1: 2$, which is very close to the XPS deduced value (0.348:0.652). For the $\mathrm{MoS}_{2}\left[\mathrm{O}_{2}\right]_{x}+2 \mathrm{O}_{\mathrm{S}}$ structure, the atomic percentage of $\mathrm{O}$ in the chemical form of $\mathrm{MoO}_{3}$ and $\mathrm{O}_{2}$ is 1:1. Therefore, the $\mathrm{MoS}_{2}\left[\mathrm{O}_{2}\right]_{x}+\mathrm{O}_{\mathrm{S}}$ model is the nearest to the real $\mathrm{MoS}_{2}\left[\mathrm{O}_{2}\right]_{x}$ superlattices we obtained by such an optimum plasma intercalation process based on the above XPS results. The calculated band structures of three representative structures including $\mathrm{MoS}_{2}$ bilayer, $\mathrm{MoS}_{2}\left[\mathrm{O}_{2}\right]_{x}$ and $\mathrm{MoS}_{2}\left[\mathrm{O}_{2}\right]_{x}+$ $\mathrm{O}_{\mathrm{S}}$ are displayed in Fig. $3 \mathrm{~g}-\mathrm{i}$, respectively, while those of other structures including $\mathrm{MoS}_{2}$ monolayer, $\mathrm{MoS}_{2}$ bilayer with substitutional $\mathrm{O}$ at $\mathrm{S}$ site $(\mathrm{Os}), \mathrm{MoS}_{2}$ bilayer with substitutional $2 \mathrm{O}$ at $2 \mathrm{~S}$ sites (2Os), and $\mathrm{MoS}_{2}$ bilayer with both oxygen molecule layer intercalated and substitutional $2 \mathrm{O}$ site at $2 \mathrm{~S}$ sites $\left(\mathrm{MoS}_{2}\left[\mathrm{O}_{2}\right]_{x}+\right.$ $2 \mathrm{O}_{\mathrm{S}}$ ), are shown in Supplementary Fig. 3.
The pristine $\mathrm{MoS}_{2}$ bilayer exhibits the interlayer distance of $6.12 \AA$ (Fig. 3a), while the interlayer distance increases slightly to 6.18 and $6.24 \AA$ for the first two cases (Fig. $3 b-c$ ) with chemically bonded oxygen atoms (Mo-O bonds). Such an increase of the interlayer distance is not only inconsistent with the above experimental results but is also insufficient to cause an indirectto-direct bandgap transition leading to the strong enhancement in PL intensity. Indeed, the calculated band structures of the first three cases (pristine $\mathrm{MoS}_{2}$ bilayer, $\mathrm{MoS}_{2}$ bilayer with $\mathrm{O}_{\mathrm{S}}$, and $\mathrm{MoS}_{2}$ bilayer with $2 \mathrm{O}_{\mathrm{S}}$ ) exhibit an indirect bandgap as shown in Fig. 3g, Supplementary Fig. 3b, c. In contrast, for the $\mathrm{MoS}_{2}\left[\mathrm{O}_{2}\right]_{x}$, $\mathrm{MoS}_{2}\left[\mathrm{O}_{2}\right]_{x}+\mathrm{O}_{S}$, and even $\mathrm{MoS}_{2}\left[\mathrm{O}_{2}\right]_{x}+2 \mathrm{O}_{\mathrm{S}}$ modes, the interlayer distance can increase up to $9.23,9.12$, and $8.96 \AA$, respectively, due to the intercalation of oxygen molecular layer, fully complying with the above experimental results. Moreover, the calculated energy band structures of all the three modes display a direct bandgap (Fig. 3h, i and Supplementary Fig. 3d), which is comparable to that of $\mathrm{MoS}_{2}$ monolayer (Supplementary Fig. 3a), further supporting the conclusion that our soft oxygen plasma intercalation indeed can produce $\mathrm{MoS}_{2}\left[\mathrm{O}_{2}\right]_{x}$ superlattice in which $\mathrm{MoS}_{2}$ monolayers alternate with oxygen molecular layers.

Figure $4 \mathrm{a}, \mathrm{b}$ presents the time-dependent PL and Raman properties of the $4 \mathrm{~L} M^{2} S_{2}$ flake with the oxygen plasma treatment. One can deduce that the optimum treatment time for oxygen intercalation is $3 \mathrm{~min}$. Once beyond this optimum treatment time, the crystal quality degrades significantly as reflected by both PL and Raman spectra. Similarly, the $6 \mathrm{~L} \mathrm{MoS}_{2}$ flake displays the same evolution with an optimum treatment time of $4 \mathrm{~min}$ as reflected by the time-dependent PL and Raman properties in Supplementary Fig. 2. In order to have a more detailed understanding of the oxygen intercalation behavior, we chose one of the most common TMD materials, CVD-grown $\mathrm{MoS}_{2}$ bilayer, and recorded the time-dependent PL intensity mapping of peak A in Fig. 4c-h. The PL intensity first decreases at the first $30 \mathrm{~s}$ and then increases drastically and reaches a maximum (20 times as high as the pristine intensity) at $60 \mathrm{~s}$. However, with the further increase in treatment time, the PL intensity decreases gradually.

By combining both the above experimental results and the DFT simulation, we obtain a clear physical picture of the oxygen plasma intercalation process as schematically illustrated in Fig. 4i. At the very beginning of the plasma treatment, the plasma irradiation can produce a small number of sulfur vacancies as well as the resultant formation of a small amount of Mo-O bonds via oxygen substitution at sulfur vacancies, thus suppressing the $\mathrm{PL}^{33}$. Thereafter, the $\mathrm{O}_{2}^{+}$ions generated by the plasma ionization (Eq. (1)) enter into the interlayer space driven by the force of the electrostatic field parallel to the interlayer space and subsequently form stable $\mathrm{O}_{2}$ molecules (Eq. (2)) there via the van der Waals interactions with the adjacent $\mathrm{MoS}_{2}$ monolayers. Consequently, the interlayer distance is expanding, effectively isolating the $\mathrm{MoS}_{2}$ and leading to the formation of $\mathrm{MoS}_{2}\left[\mathrm{O}_{2}\right]_{x}$ superlattice with intriguing PL comparable with that of the corresponding intrinsic monolayer. Obviously, the interspace expansion effect prevails over the influence of plasma-induced Mo-O bonds at the optimum treatment time. However, once the treatment time is beyond this critical value, the influence of the plasma-induced Mo-O bonds may become more and more prominent, finally giving rise to the degradation of the crystal quality as reflected by both PL and Raman spectra. The time-dependent PL intensity mapping studies on vertically oriented $\mathrm{MoS}_{2}$ bilayer (Supplementary Fig. 4) shows no clue of any intercalation effect but the PL degradation, further proving the determining role of the parallel electrostatic field as well as the influence of plasmainduced Mo-O bonds. As shown in Supplementary Fig. 5, the 
a

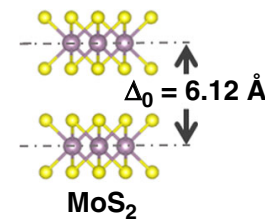

d

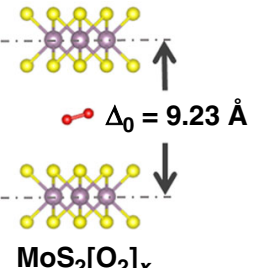

b

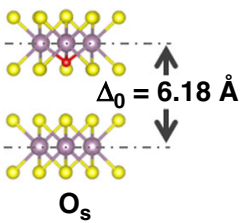

e
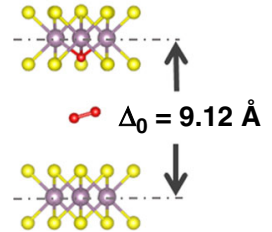

$\operatorname{MoS}_{2}\left[\mathrm{O}_{2}\right]_{x}+\mathrm{O}_{\mathrm{s}}$
C 98890

$\begin{aligned} \Delta_{0} & =6.24 \AA\end{aligned}$

$-.8820 \downarrow$

$2 \mathrm{O}_{\mathrm{s}}$

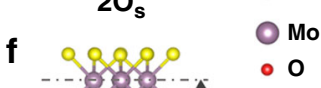

0
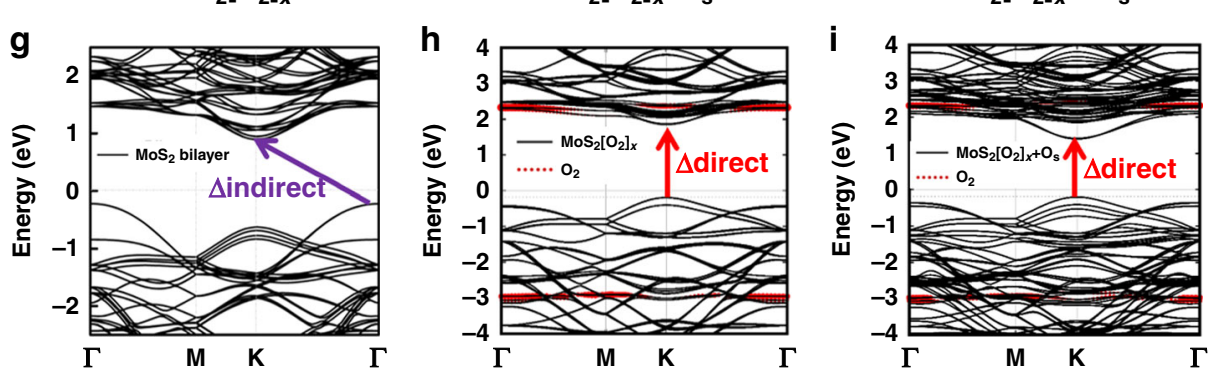

Fig. 3 Calculated atomic configurations and energy band structures confirm interlayer space expansion and transition to direct optical bandgap. a-e Calculated atomic configurations of a the pristine $\mathrm{MoS}_{2}$ bilayer and four oxygen-involved $\mathrm{MoS}_{2}$ bilayer systems, namely $\mathrm{MoS}_{2}$ bilayer $\mathbf{b}$ with substitutional $\mathrm{O}$ site at $\mathrm{S}$ site $\left(\mathrm{O}_{\mathrm{S}}\right)$, c with substitutional $2 \mathrm{O}$ at $2 \mathrm{~S}$ sites $\left(2 \mathrm{O}_{\mathrm{S}}\right)$, d with oxygen molecule layer intercalated $\left(\mathrm{MoS}_{2}\left[\mathrm{O}_{2}\right]_{x}\right)$, e with both oxygen molecule layer intercalated and substitutional $\mathrm{O}$ site at $\mathrm{S}$ site $\left(\mathrm{MoS}_{2}\left[\mathrm{O}_{2}\right]_{x}+\mathrm{O}_{\mathrm{S}}\right)$, f with both oxygen molecule layer intercalated and substitutional $2 \mathrm{O}$ site at $2 \mathrm{~S}$ sites $\left(\mathrm{MoS}_{2}\left[\mathrm{O}_{2}\right]_{x}+2 \mathrm{O}_{\mathrm{S}}\right)$. g-i Calculated band structures of the pristine $\mathrm{MoS}_{2}$ bilayer $(\mathbf{f})$, the $\mathrm{MoS}_{2}\left[\mathrm{O}_{2}\right]_{x}$ structure $(\mathbf{g})$, and the $\mathrm{MoS}_{2}\left[\mathrm{O}_{2}\right]_{x}+\mathrm{O}_{\mathrm{S}}$ structure, in which the atomic percentage of $\mathrm{O}$ in the chemical form of $\mathrm{MoO}_{3}$ and $\mathrm{O}_{2}$ is 1:2 (close to the XPS deduced ratio 0.348:0.652). The red dotted line in $\mathbf{g}$ corresponds to the energy state of the oxygen molecule.

$\mathrm{MoS}_{2}\left[\mathrm{O}_{2}\right]_{x}$ superlattices based on bilayer, three-layer, four-layer, and six-layer $\mathrm{MoS}_{2}$ flakes exhibit good stability by maintaining its intriguing PL properties over 30 days.

Photoelectric properties of $\mathrm{MoS}_{2}\left[\mathrm{O}_{2}\right]_{x} / \mathrm{WS}_{2}\left[\mathrm{O}_{2}\right]_{x}$ superlattices. We have also studied the photoelectrical properties of the same $\mathrm{MoS}_{2} / \mathrm{WS}_{2}$ bilayer heterostructure and $\mathrm{MoS}_{2}\left[\mathrm{O}_{2}\right]_{x} / \mathrm{WS}_{2}\left[\mathrm{O}_{2}\right]_{x}$ superlattice heterostructure before and after the plasma intercalation process. Figure $5 \mathrm{a}, \mathrm{b}$ displays the schematic illustration and the output characteristic $\left(I_{\mathrm{ds}}-V_{\mathrm{ds}}\right.$ curves $)$ of the $\mathrm{MoS}_{2}\left[\mathrm{O}_{2}\right]_{x} /$ $\mathrm{WS}_{2}\left[\mathrm{O}_{2}\right]_{x}$ superlattice heterostructure under the $532 \mathrm{~nm}$ laser illumination with different power intensities, respectively. The photocurrent $\left(I_{\mathrm{ds}}\right)$ increases with the increase of the incident power, indicating that the number of photo-induced carriers increases as a result of the increase in the number of absorbed photons. The energy band diagram of such superlattice heterostructure shown in the inset of Fig. 5b exhibits an extremely small build-in electric field directing from $\mathrm{MoS}_{2}\left[\mathrm{O}_{2}\right]_{x}$ to $\mathrm{WS}_{2}\left[\mathrm{O}_{2}\right]_{x}$ due to their similar bandgap $\left(1.87 \mathrm{eV}\right.$ for $\mathrm{MoS}_{2}\left[\mathrm{O}_{2}\right]_{x}$ and $1.97 \mathrm{eV}$ for $\left.\mathrm{WS}_{2}\left[\mathrm{O}_{2}\right]_{\mathrm{x}}\right)$ and identical n-type characteristics. This extremely small build-in electric field can be clearly reflected by the fact that the photocurrent $\left(I_{\mathrm{ds}}\right)$ exhibits a faster increase with the increase in laser intensity under $V_{\mathrm{ds}}>0 \mathrm{~V}$ (forward bias) compared to the case under $V_{\mathrm{ds}}<0 \mathrm{~V}$ (reverse bias). Therefore, we choose two representative cases, the reverse bias $\left(V_{\mathrm{ds}}=-1 \mathrm{~V}\right)$ and the forward bias $\left(V_{\mathrm{ds}}=1 \mathrm{~V}\right)$ to compare the photoelectrical properties between the same $\mathrm{MoS}_{2} / \mathrm{WS}_{2}$ bilayer heterostructure (before the plasma intercalation) and $\mathrm{MoS}_{2}\left[\mathrm{O}_{2}\right]_{x} / \mathrm{WS}_{2}\left[\mathrm{O}_{2}\right]_{x}$ superlattice heterostructure (after the optimum plasma intercalation), as shown in Fig. $5 c$, d, respectively. For the former case $\left(V_{\mathrm{ds}}=-1 \mathrm{~V}\right)$, the on/off ratio increases from $\sim 10$ to $\sim 70$ and the photocurrent increases by 70 times from 0.04 to $2.83 \mathrm{nA}$ after the plasma intercalation process. For the latter case $\left(V_{\mathrm{ds}}=1 \mathrm{~V}\right)$, the photocurrent increases by more than 100 times from 0.46 to $48.7 \mathrm{nA}$ after the plasma intercalation process although the on/off ratio is hard to estimate due to the longer decaying time. This strikingly enhanced photoresponse can be attributed to the indirect-todirect energy band transition after the plasma intercalation process $^{34,35}$. We also fabricated a vertical $3 \mathrm{~L}-\mathrm{MoS}_{2} /$ multilayer$\mathrm{WSe}_{2} \mathrm{p}-\mathrm{n}$ heterojunction by the mechanical exfoliation and transfer method. The optimum plasma intercalation process can translate the top $3 \mathrm{~L} \mathrm{MoS}_{2}$ flake with an indirect bandgap into $\mathrm{MoS}_{2}\left[\mathrm{O}_{2}\right]_{x}$ superlattice with a direct bandgap and thus make the resultant self-powered photocurrent at $V_{\mathrm{ds}}=0 \mathrm{~V}$ largely increased by 20 times (Supplementary Fig. 6), suggesting that our soft plasma intercalation technique has good stability and reproducibility in controlling the optical and electronic properties of these superlattices. The photoelectric performance can be further improved by using thicker superlattice lateral heterostructures with higher absorption.

Our approach may be expanded to intercalate diverse 2D mechanically exfoliated or CVD-grown TMD flakes, including $\mathrm{WS}_{2}, \mathrm{MoSe}_{2}$, and $\mathrm{ReS}_{2}$, etc. with thicknesses ranging from 2 to 8 layers, as evidenced by Supplementary Figs. 7-9, respectively. Similarly to the $\operatorname{MoS}_{2}\left[\mathrm{O}_{2}\right]_{x}$ superlattice, $\mathrm{WS}_{2}\left[\mathrm{O}_{2}\right]_{x}$ and $\mathrm{MoSe}_{2}\left[\mathrm{O}_{2}\right]_{x}$ superlattices exhibit the same variation trend in the structural and optical properties compared to their pristine counterparts. However, for $\operatorname{ReS}_{2}\left[\mathrm{O}_{2}\right]_{x}$ superlattice, no bandgap transition can be observed apart from the interlayer expansion. This result can be ascribed to the fact that both $\mathrm{ReS}_{2}$ monolayer and multilayer are direct bandgap semiconductors ${ }^{36-38}$. By considering the determining role of the electric field parallel to the interlayer space of TMDs, we expect that proper alignment of the plasma generated electric fields may lead to the observation of 
a

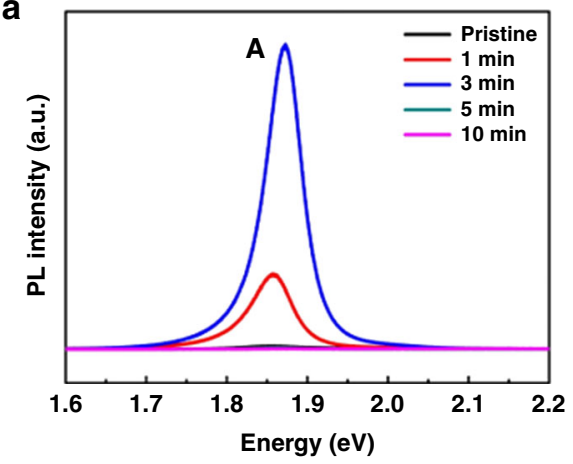

b

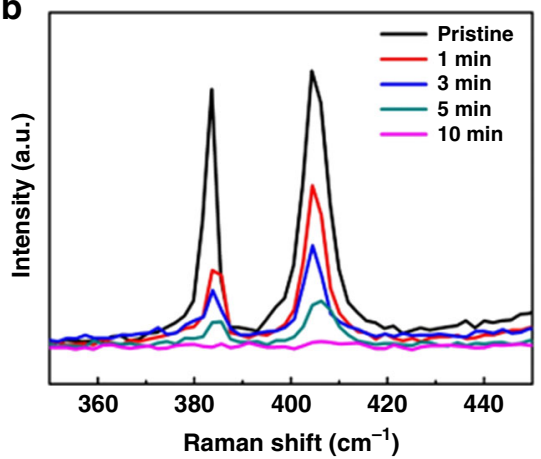

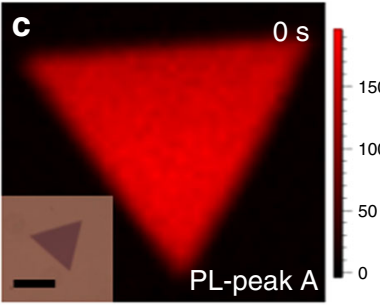
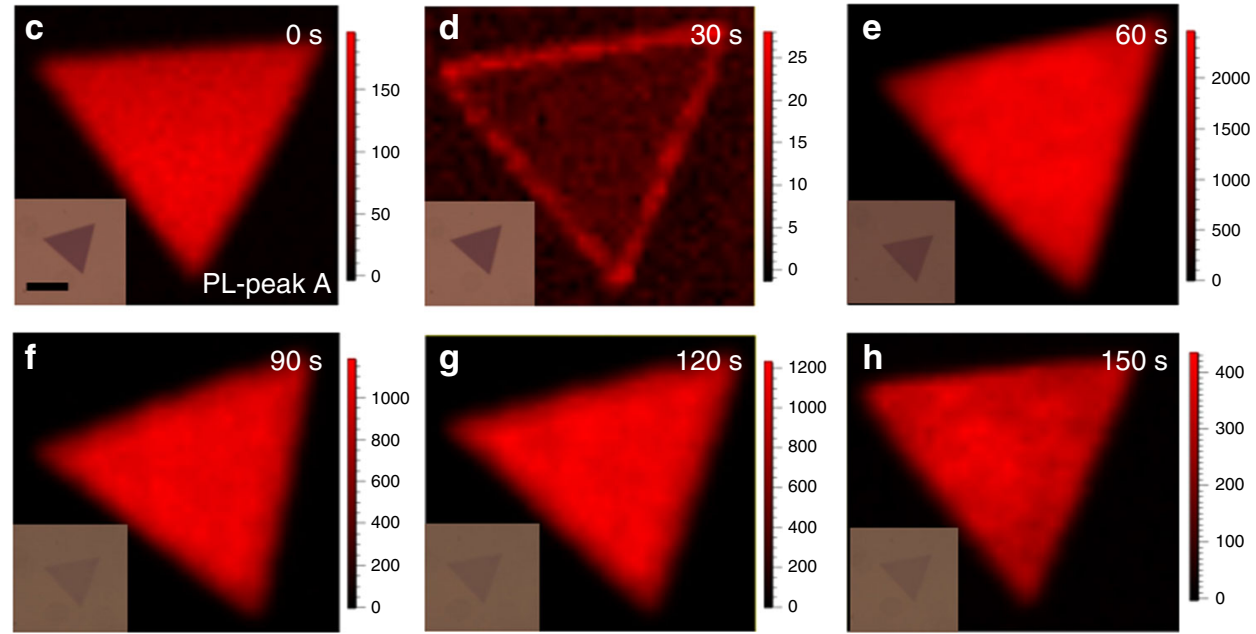

i
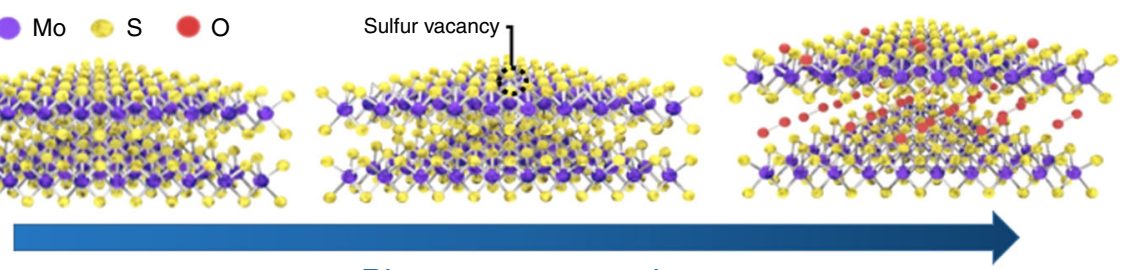

Plasma treatment time

Fig. 4 Treatment time-dependent PL and Raman properties and mechanism of the oxygen plasma intercalation. $\mathbf{a}, \mathbf{b}$ Time-dependent PL (a) and Raman (b) spectra of the plasma-treated $4 \mathrm{~L} \mathrm{MoS}_{2}$ flake. c-h Time-dependent PL intensity mapping of peak $\mathrm{A}$ of the plasma-treated CVD-grown MoS 2 bilayer flake: c 0 s, d 30 s, e 60 s, f 90 s, g 120 s, and h 150 s. i Schematic physical picture of the oxygen plasma intercalation process.

similar oxygen intercalation effects in other types of lowtemperature plasmas.

\section{Discussion}

In summary, the presented soft oxygen plasma intercalation generates 2D ACMSs where TMD monolayers alternate with oxygen molecular layers. This dry method is suitable for mechanically exfoliated or CVD-grown TMD flakes (including $\mathrm{MoS}_{2}, \mathrm{WS}_{2}, \mathrm{MoSe}_{2}$, and $\mathrm{ReS}_{2}$, etc.) with thicknesses ranging from 2 to 8 layers. The physical mechanism can be ascribed to the $\mathrm{O}_{2}{ }^{+}$ ions entering the interlayer space driven by the parallel electric field and then forming stable $\mathrm{O}_{2}$ molecules via van der Waals interactions with the adjacent TMD monolayers. The interlayer distance can be largely expanded and becomes sufficient to effectively isolate the TMD monolayers as well as to almost suppress the interlayer coupling. These effects make superlattices such as $\mathrm{MoS}_{2}\left[\mathrm{O}_{2}\right]_{x}, \mathrm{WS}_{2}\left[\mathrm{O}_{2}\right]_{x}, \mathrm{MoSe}_{2}\left[\mathrm{O}_{2}\right]_{x}$, and $\mathrm{ReS}_{2}\left[\mathrm{O}_{2}\right]_{x}$ display monolayer characteristics. The bilayer $\operatorname{MoS}_{2}\left[\mathrm{O}_{2}\right]_{x} / \mathrm{WS}_{2}\left[\mathrm{O}_{2}\right]_{x}$ superlattice lateral heterostructures show much better photoelectric performance (100 times increased photocurrent) than the pristine bilayer $\mathrm{MoS}_{2} / \mathrm{WS}_{2}$ lateral heterostructures because of the indirect-to-direct energy band transition. Our studies thus provide a potentially universal approach to create such $2 \mathrm{D}$ ACMSs from pristine $2 \mathrm{D}$ nanomaterials and provide a generic platform for fundamental physics research and potential technological applications. Moreover, the 2D ACMSs with intrinsic monolayer characteristics such as a direct optical bandgap set an important milestone for future optoelectronics.

\section{Methods}

Sample preparation. Few-layer $\mathrm{MoS}_{2}, \mathrm{WS}_{2}, \mathrm{MoSe}_{2}$, and $\mathrm{ReS}_{2}$ flakes were exfoliated mechanically from corresponding bulk single crystals and deposited onto $300 \mathrm{~nm} \mathrm{SiO} / 2 / \mathrm{Si}$ substrates. Bilayer $\mathrm{MoS}_{2}$ flakes were synthesized on $300 \mathrm{~nm} \mathrm{SiO} / 2 \mathrm{Si}$ substrates by chemical vapor epitaxy. Before mechanical exfoliation or CVD, all the substrates were first ultrasonically cleaned in acetone and alcohol, and then rinsed in deionized water and finally dried by nitrogen stream.

Oxygen plasma intercalation. As shown in Supplementary Fig. 1, a home-made planar low-frequency $(2 \mathrm{MHz})$ inductively coupled plasma system was applied to produce the $\mathrm{MoS}_{2}\left[\mathrm{O}_{2}\right]_{x}, \mathrm{WS}_{2}\left[\mathrm{O}_{2}\right]_{x}, \mathrm{MoSe}_{2}\left[\mathrm{O}_{2}\right]_{x}$, and $\operatorname{ReS}_{2}\left[\mathrm{O}_{2}\right]_{x}$ superlattice. The input RF power was kept at as low as $20 \mathrm{~W}$ so that the plasma was excited in the Emode of ICP, producing a radial electrostatic field parallel to the substrate surface. 
a
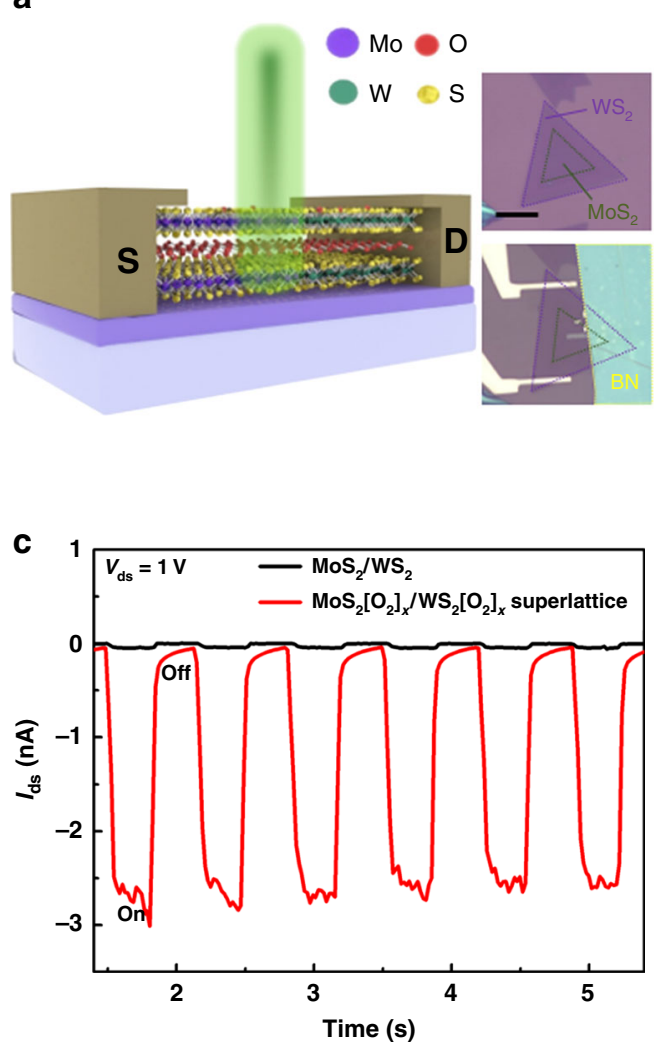

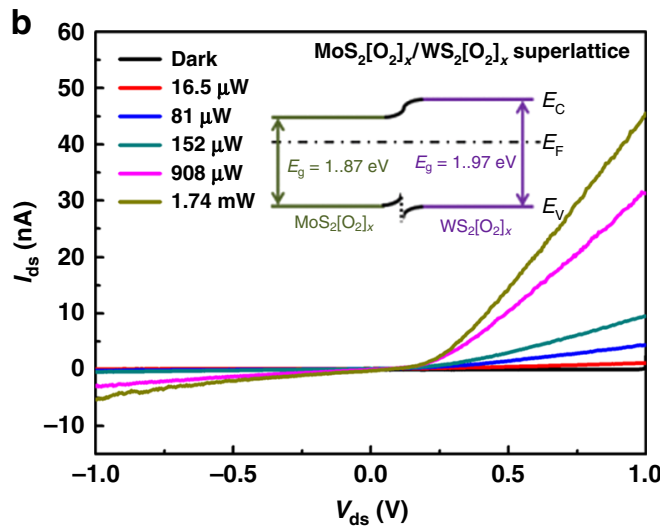

d

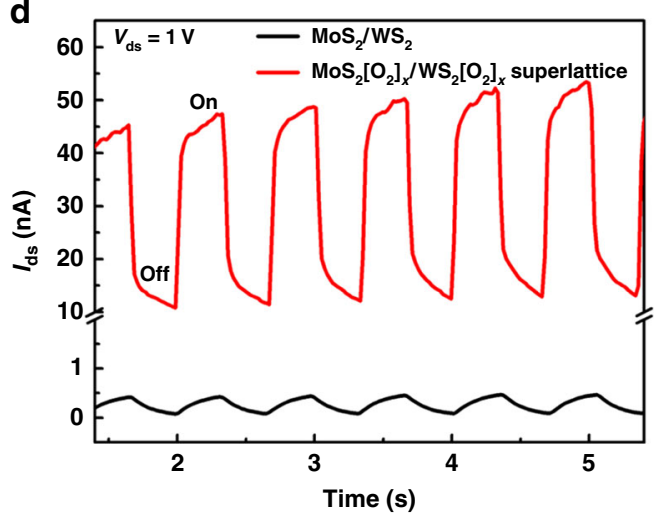

Fig. 5 Evolution of photoelectric properties from MoS2/WS2 bilayer heterostructure to $\mathbf{M o S}_{\mathbf{2}}\left[\mathrm{O}_{\mathbf{2}}\right]_{\mathbf{x}} / \mathrm{WS}_{\mathbf{2}}\left[\mathrm{O}_{\mathbf{2}}\right]_{\mathbf{x}}$ superlattice heterostructure.

a Schematic illustration and typical optical images of the $\operatorname{MoS}_{2}\left[\mathrm{O}_{2}\right]_{x} / \mathrm{WS}_{2}\left[\mathrm{O}_{2}\right]_{x}$ superlattice heterostructure. $\mathbf{b} I_{\mathrm{ds}}-V_{\mathrm{ds}}$ curves of the $\mathrm{MoS}_{2}\left[\mathrm{O}_{2}\right]_{x} /$ $\mathrm{WS}_{2}\left[\mathrm{O}_{2}\right]_{x}$ superlattice heterostructure photodetector under $532 \mathrm{~nm}$ laser illumination with different power intensities. Inset is the energy band diagram of the $\mathrm{MoS}_{2}\left[\mathrm{O}_{2}\right]_{x} / \mathrm{WS}_{2}\left[\mathrm{O}_{2}\right]_{x}$ superlattice heterostructure. c, $\mathbf{d}$ The time-resolved photoresponse of both $\mathrm{MoS}_{2} / \mathrm{WS}_{2}$ bilayer heterostructure (before the plasma intercalation) and corresponding $\mathrm{MoS}_{2}\left[\mathrm{O}_{2}\right]_{x} / \mathrm{WS}_{2}\left[\mathrm{O}_{2}\right]_{x}$ superlattice heterostructure (after the optimum plasma intercalation) under $\mathbf{c} V_{\mathrm{ds}}=-1 \mathrm{~V}$ (reverse bias) and $\mathbf{d} V_{\mathrm{ds}}=1 \mathrm{~V}$ (forward bias) with $532 \mathrm{~nm}(1.74 \mathrm{~mW}$ ) illumination.

The working pressure was kept at $38 \mathrm{~Pa}$ by introducing $\mathrm{O}_{2}$ as the precursor gas with a flow rate of $10 \mathrm{sccm}$. The sample stage was kept rotating during the plasma treatment process to ensure uniform intercalation into the interlayer space of TMD flakes. Unless other specified, the samples were placed horizontally on the sample stage.

Characterizations. Optical images were obtained by Optical Microscopy (Leica $4000 \mathrm{M}$ or Leica $2700 \mathrm{M})$. AFM was carried out by using a Bruker Dimension ICON system in the tapping mode. Raman and PL measurements were recorded using a Renishaw Invia micro-Raman spectrometer with a $532 \mathrm{~nm}$ excitation laser. The laser power was lower than $0.2 \mathrm{~mW}$ to avoid any laser-induced heating. Raman spectra were measured using an $1800 \mathrm{l} / \mathrm{mm}$ grating to disperse the signal while PL ones were measured using a $600 \mathrm{l} / \mathrm{mm}$ grating. XPS (Thermo Scientific Esca lab 250Xi) with an $\mathrm{Al}-\mathrm{Ka}(1486.6 \mathrm{eV})$ source was used to determine the chemical configurations of the TMD flake before and after oxygen plasma intercalation. The cross-sectional high-resolution transmission electron microscopy images were measured by using a Themis z TEM system with an accelerating voltage of $200 \mathrm{kV}$. All the measurements were performed at room temperature under ambient conditions.

Device fabrication and photoelectrical measurements. Bilayer $\mathrm{MoS}_{2} / \mathrm{WS}_{2}$ lateral heterojunction flakes were synthesized on $300 \mathrm{~nm} \mathrm{SiO} / 2 / \mathrm{Si}$ substrates by adjusting both the temperature and the carrier gas flow direction. Then, the exfoliated BN flakes were transferred onto the surface of bilayer $\mathrm{MoS}_{2} / \mathrm{WS}_{2}$ heterojunction flakes serving as an insulating layer via a dry transfer method with polydimethylsiloxane (PDMS). Subsequently, we fabricated $\mathrm{Cr} / \mathrm{Au}(5 \mathrm{~nm} / 50 \mathrm{~nm})$ contact electrodes by using e-beam lithography and electron beam evaporation. After that, the bilayer $\mathrm{MoS}_{2} / \mathrm{WS}_{2}$ heterojunction devices were subjected to soft oxygen plasma intercalation and transformed into $\operatorname{MoS}_{2}\left[\mathrm{O}_{2}\right]_{x} / \mathrm{WS}_{2}\left[\mathrm{O}_{2}\right]_{x}$ superlattice heterostructures. The electrical and photoresponse characteristics of the same $\mathrm{MoS}_{2} / \mathrm{WS}_{2}$ bilayer heterostructure and $\mathrm{MoS}_{2}\left[\mathrm{O}_{2}\right]_{x} / \mathrm{WS}_{2}\left[\mathrm{O}_{2}\right]_{x}$ superlattice heterostructure before and after the plasma intercalation process were measured using a Keithley 2643B analyzer under dark and illuminated conditions in an atmospheric environment. Renishaw Invia micro-Raman spectrometer with a
$532 \mathrm{~nm}$ excitation laser was employed to attain the spectral photocurrent response. For all the photocurrent measurements, the lasers were focused on the sample with a $50 \times$ objective $(\mathrm{NA}=0.5)$ and the spot size of the light is about $1 \mu \mathrm{m}$, much smaller than the device channel length. Optical attenuators were used to change the power of the illuminated laser and a chopper with a frequency of $1 \mathrm{~Hz}$ was used to record the time-dependent photoresponse.

First-principles calculations. The first-principles calculations were performed by the Vienna ab initio simulation package ${ }^{39}$ and the projected augmented-wave potential ${ }^{40,41}$. The exchange-correlation functional introduced by Perdew, Burke, and Ernzerhof ${ }^{42}$ within the generalized gradient approximation was applied in the calculations. The $p$ semi-core states of Mo were described as valence electrons. For bilayer $\mathrm{MoS}_{2}$ we constructed a slab geometry with the insertion of a vacuum layer of $15 \AA$. The k-space mesh used was $12 \times 12 \times 1$ for the slab structure. The energy cutoff for the plane-wave basis was set as $520 \mathrm{eV}$ and the forces are relaxed less than $0.01 \mathrm{eV} / \mathrm{A}$. The positions of atoms were allowed to relax while the lattice constants of the unit cells were fixed to the experimental values.

\section{Data availability}

The data that support the findings of this study are available from the corresponding author on reasonable request.

Received: 6 February 2020; Accepted: 22 October 2020; Published online: 24 November 2020

\section{References}

1. Novoselov, K. S., Mishchenko, A., Carvalho, A. \& Castro Neto, A. H. 2D materials and van der Waals heterostructures. Science 353, 9439 (2016).

2. Liu, Y. et al. Van der Waals heterostructures and devices. Nat. Rev. Mater. 1, 16042 (2016). 
3. Jariwala, D., Marks, T. J. \& Hersam, M. C. Mixed-dimensional van der Waals heterostructures. Nat. Mater. 16, 170-181 (2016).

4. Wang, C. et al. Monolayer atomic crystal molecular superlattices. Nature 555, 231-236 (2018).

5. Ryu, Y. K., Frisenda, R. \& Castellanos-Gomez, A. Superlattices based on van der Waals 2D materials. Chem. Commun. 55, 11498-11510 (2019).

6. Dean, C. R. et al. Boron nitride substrates for high-quality graphene electronics. Nat. Nanotech. 5, 722-726 (2010).

7. Withers, F. et al. Light-emitting diodes by band-structure engineering in van der Waals heterostructures. Nat. Mater. 14, 301-306 (2015).

8. Haigh, S. J. et al. Cross-sectional imaging of individual layers and buried interfaces of graphene-based heterostructures and superlattices. Nat. Mater. 11, 764-767 (2012).

9. $\mathrm{Yu}, \mathrm{W}$. J. et al. Vertically stacked multi-heterostructures of layered materials for logic transistors and complementary inverters. Nat. Mater. 12, 246-252 (2013).

10. Cheng, R. et al. Electroluminescence and photocurrent generation from atomically sharp $\mathrm{WSe}_{2} / \mathrm{MoS}_{2}$ heterojunction p-n diodes. Nano Lett. 14, 5590-5597 (2014).

11. Lee, C. H. et al. Atomically thin p-n junctions with van der Waals heterointerfaces. Nat. Nanotech. 9, 676-681 (2014).

12. Duan, X. D. et al. Lateral epitaxial growth of two-dimensional layered semiconductor heterojunctions. Nat. Nanotech. 9, 1024-1030 (2014).

13. Gong, Y. J. et al. Vertical and in-plane heterostructures from $\mathrm{WS}_{2} / \mathrm{MoS}_{2}$ monolayers. Nat. Mater. 13, 1135-1142 (2014).

14. Yan, A. M. et al. Direct growth of single- and few-layer $\mathrm{MoS}_{2}$ on h-BN with preferred relative rotation angles. Nano Lett. 15, 6324-6331 (2015).

15. Zhang, Z. W. et al. Robust epitaxial growth of two-dimensional heterostructures, multiheterostructures, and superlattices. Science 357, 788-792 (2017).

16. Li, M. Y. et al. Epitaxial growth of a monolayer $\mathrm{WSe}_{2}-\mathrm{MoS}_{2}$ lateral p-n junctionwith an atomically sharp interface. Science 349, 524-528 (2015).

17. Zhang, X. M. et al. Transition metal dichalcogenides bilayer single crystals by reverse-flow chemical vapor epitaxy. Nat. Commun. 10, 598 (2019).

18. Bao, W. Z. et al. Approaching the limits of transparency and conductivity in graphitic materials through lithium intercalation. Nat. Commun. 5, 4224 (2014).

19. Xiong, F. et al. Li intercalation in $\mathrm{MoS}_{2}$ : in situ observation of its dynamics and tuning optical and electrical properties. Nano Lett. 15, 6777-6784 (2015).

20. Yu, Y. J. et al. Gate-tunable phase transitions in thin flakes of $1 \mathrm{~T}-\mathrm{TaS}_{2}$. Nat. Nanotech. 10, 270-276 (2015).

21. Lin, Y. et al. Dielectric screening of excitons and trions in single-layer $\mathrm{MoS}_{2}$. Nano Lett. 14, 5569-5576 (2014)

22. Xie, Y. et al. Controllable growth of monolayer $\mathrm{MoS}_{2}$ by chemical vapor deposition via close $\mathrm{MoO}_{2}$ precursor for electrical and optical applications. Nanotechnology 28, 084001 (2017).

23. Splendiani, A. et al. Emerging photoluminescence in monolayer $\mathrm{MoS}_{2}$. Nano Lett. 10, 1271-1275 (2010)

24. Li, H. et al. From bulk to monolayer $\mathrm{MoS}_{2}$ : evolution of raman scattering. $\mathrm{Adv}$. Funct. Mater. 22, 1385-1390 (2012).

25. Lee, C. et al. Anomalous lattice vibrations of single- and few-layer $\mathrm{MoS}_{2}$. ACS Nano 4, 2695-2700 (2010).

26. Dhall, R. et al. Direct bandgap transition in many-layer $\mathrm{MoS}_{2}$ by plasmainduced layer decoupling. Adv. Mater. 27, 1573-1578 (2015).

27. Mak, K. F., Lee, C., Hone, J., Shan, J. \& Heinz, T. F. Atomically thin $\mathrm{MoS}_{2}$ : a new direct-gap semiconductor. Phys. Rev. Lett. 105, 136805 (2010).

28. Kim, S. et al. Effects of plasma treatment on surface properties of ultrathin layered $\mathrm{MoS}_{2}$. 2D Mater. 3, 035002 (2016).

29. Petersson, L. G. \& Karlsson, S. E. Clean and oxygen exposed potassium studied by photoelectron spectroscopy. Phys. Scr. 16, 425-431 (1977).

30. Brown, N. M. D., Cui, N. Y. \& Mckinley, A. An XPS study of the surface modification of natural $\mathrm{MoS}_{2}$ following treatment in an RF-oxygen plasma. Appl. Surf. Sci. 134, 11-21 (1998).

31. Wu, S. X. et al. High-performance p-type $\mathrm{MoS}_{2}$ field-effect transistor by toroidal-magnetic-field controlled oxygen plasma doping. $2 D$ Mater. 6, 025007 (2019).

32. Tang, B. et al. Direct n- to $\mathrm{p}$-type channel conversion in monolayer/few-Layer $\mathrm{WS}_{2}$ field-effect transistors by atomic nitrogen treatment. ACS Nano 12, 2506-2513 (2018).

33. Kang, N., Paudel, H. P., Leuenberger, M. N., Tetard, L. \& Khondaker, S. I. Photoluminescence quenching in single-layer $\mathrm{MoS}_{2}$ via oxygen plasma treatment. J. Phys. Chem. C 118, 21258-21263 (2014).

34. Wang, Q. H., Kalantar-Zadeh, K., Kis, A., Coleman, J. N. \& Strano, M. S. Electronics and optoelectronics of two-dimensional transition metal dichalcogenides. Nat. Nanotechnol. 7, 699-712 (2012).
35. Lei, J. et al. Layer-dependent photoresponse of $2 \mathrm{D} \mathrm{MoS}_{2}$ films prepared by pulsed laser deposition. J. Mater. Chem. C 7, 2522-2529 (2019).

36. Shim, J. et al. Thin-film transistors: high-performance 2D rhenium disulfide $\left(\mathrm{ReS}_{2}\right)$ transistors and photodetectors by oxygen plasma treatment. $A d v$. Mater. 28, 6984-6984 (2016).

37. Rahman, M., Davey, K. \& Qiao, S. Z. Advent of $2 \mathrm{D}$ rhenium disulfide $\left(\mathrm{ReS}_{2}\right)$ fundamentals to applications. Adv. Funct. Mater. 27, 1606129 (2017).

38. Hafeez, M., Gan, L., Li, H. Q., Ma, Y. \& Zhai, T. Y. Large-area bilayer $\mathrm{ReS}_{2}$ film/multilayer $\mathrm{ReS}_{2}$ flakes synthesized by chemical vapor deposition for high performance photodetectors. Adv. Funct. Mater. 26, 4551-4560 (2016).

39. Huang, Q. et al. First-principles study of O-BN: A sp ${ }^{3}$-bonding boron nitride allotrope. J. Appl. Phys. 112, 053518 (2012).

40. Lee, B. \& Rudd, R. E. First-principles calculation of mechanical properties of Si $\langle 001\rangle$ nanowires and comparison to nanomechanical theory. Phys. Rev. B 75, 195328 (2007)

41. Kresse, G. \& Joubert, D. From ultrasoft pseudopotentials to the projector augmented-wave method. Phys. Rev. B 59, 1758-1775 (2007).

42. Perdew, J. P., Burke, K. \& Ernzerhof, M. Generalized gradient approximation made simple. Phys. Rev. Lett. 77, 3865-3868 (1996).

\section{Acknowledgements}

This work is partially supported by the National Nature Science Foundation under Grants 62074070, 11704159, and 11574215, the Natural Science Foundation of Jiangsu Province, China under Grants BK20170167 and BK20190576, the Fundamental Research Funds for the Central Universities of China under Grants JUSRP51726B, the 111 Project under Grant B12018, Postgraduate Research \& Practice Innovation Program of Jiangsu Province under Grant KYCX18_1860. A.J.D. and K.O. acknowledge partial support by the Australian Research Council (ARC).

\section{Author contributions}

S.Q.X. conceived the project, designed the experiment, and jointly with K.O. conceptualized the ideas. L.F.Z. and H.Y.N. performed the experiment. X.M.Z. performed the CVD synthesis of TMD flakes and heterojunctions. Q.F.L. and A.J.D. provided theoretical support. L.F.Z. supervised by H.Y.N. and Z.H.N. measured the photoresponse S.Q.X., L.F.Z., H.Y.N., Q.F.L., X.F.G., and K.O. jointly analyzed the data and interpreted the results. S.Q.X., L.F.Z., H.Y.N., K.O., and X.F.G. co-wrote the paper. All co-authors discussed the results and commented on the paper at all stages.

\section{Competing interests}

The authors declare no competing interests.

\section{Additional information}

Supplementary information is available for this paper at https://doi.org/10.1038/s41467 020-19766-x.

Correspondence and requests for materials should be addressed to S.X.

Peer review information Nature Communications thanks the anonymous reviewer(s) for their contribution to the peer review of this work.

Reprints and permission information is available at http://www.nature.com/reprints

Publisher's note Springer Nature remains neutral with regard to jurisdictional claims in published maps and institutional affiliations.

Open Access This article is licensed under a Creative Commons Attribution 4.0 International License, which permits use, sharing, adaptation, distribution and reproduction in any medium or format, as long as you give appropriate credit to the original author(s) and the source, provide a link to the Creative Commons license, and indicate if changes were made. The images or other third party material in this article are included in the article's Creative Commons license, unless indicated otherwise in a credit line to the material. If material is not included in the article's Creative Commons license and your intended use is not permitted by statutory regulation or exceeds the permitted use, you will need to obtain permission directly from the copyright holder. To view a copy of this license, visit http://creativecommons.org/ licenses/by/4.0/

(C) The Author(s) 2020 